\title{
Scattering by a Plane Interface That Contains Strongly Conducting Patches
}

\author{
Chris J. Coleman, Member, IEEE
}

\begin{abstract}
A technique is developed for estimating the scattering effects of a homogeneous half space that contains strongly conducting patches on its surface. The technique directly relates the radiative behavior of the scattered field to that of the field sources acting alone. In particular, the method can be used to relate the performance of an antenna over a perfectly conducting plane to its performance over a finite ground screen. Unlike some previous attempts at this problem, the present approach does not rely upon an impedance boundary condition or upon a physical optics approximation to screen currents.
\end{abstract}

Index Terms-Antennas, ground screens, reciprocity, scattering.

\section{INTRODUCTION}

$\mathbf{T}$ HE SCATTERING effects of a homogeneous ground are well known through the fundamental work of Sommerfeld [1], [2] and the more practical developments of Norton [3] and King [4]. Sommerfeld derived the field of an arbitrary Hertzian dipole placed above a finitely conducting homogeneous ground. Since an arbitrary source can be represented as a combination of such dipoles, this work also laid the foundations for considering fields with far more complex sources. In particular, the Sommerfeld solution can be used as part of a procedure for analyzing the behavior of an antenna near a realistic ground [5]. While a homogeneous ground is a good approximation in many situations, it is not appropriate when the antenna is provided with a finite ground screen. It is possible, in principle, to represent the screen by a combination of dipole elements and, hence, solve the problem using a numerical simulation code such as numerical electromagnetic code (NEC) [5]. Unfortunately, due to the large number of degrees of freedom that are required, this approach will become unwieldy for anything but the smallest of screens. An alternative is to solve for the antenna currents as if over a uniform ground and then use these currents as sources for a separate investigation of the scattering effects of the screen. (Since the antenna currents are mainly influenced by conditions in the immediate vicinity of the antenna, it is sufficient to assume a uniform ground with the same properties as the ground immediately below the antenna.) One of the most important techniques for implementing this approach is based on the compensation theorem [6], [7], but alternative treatments based on the extinction theorem [8], [9] have been developed. Most approaches, however, employ a surface impedance representation of the ground and calculate the currents on the screen by

Manuscript received February 27, 2001; revised October 20, 2001.

The author is with the Electrical and Electronic Engineering Department, the University of Adelaide, SA 5005, Australia (e-mail: ccoleman @eleceng. adelaide.edu.au).

Digital Object Identifier 10.1109/TAP.2003.808529 means of a physical optics approximation. The antenna ground screen problem can be regarded as a special case of the more generic problem of relating incident and scattered fields when the scatterer consists of a plane interface containing strongly conducting patches (the ground screens). It is the purpose of the current paper to derive a suitable relationship without recourse to an impedance boundary condition or a physical optics approximation.

The reciprocity theorem of electromagnetism [10] provides a general formulation from which many important integral results can be derived (the compensation and extinction theorems for example). In particular, the theorem is applicable to situations where the medium is nonhomogeneous and, hence, can be applied to the problem of a plane interface with strongly conducting patches. By separately applying the reciprocity theorem to the regions above and below interface, the present study has derived a relationship between the radiation behavior of the field sources acting alone and the scattered radiation caused by the patches. For a general distribution of patches, and realistic limit of interface parameters, this relationship allows the scattered fields to be calculated without considering the unknown currents on the ground screen. The results are identical to those previously derived by the author [11] through a surface impedance boundary condition together with a physical optics approximation to the current on the patches. Section II of this paper develops the basic theory of the method, Section III develops a practical implementation, and Section IV considers some applications. The applications include examples with complex patch geometry and show how antenna ground screens can be used as another element in the optimization of antenna patterns.

\section{BASIC THEORY}

The problem of interest is illustrated in Fig. 1. It consists of calculating the scattered field for radiation that is incident upon a half space whose surface contains perfectly conducting patches. The incident radiation is generated by known (time harmonic) current sources and the half space has finite conductivity. (It is assumed that the conductivity is sufficiently large for surface waves to be negligible.) Across the surface that divides the upper and lower half spaces, the tangential components of the electromagnetic field $(\underline{H}, \underline{E})$ will be continuous outside the patches. Within the patches, however, the tangential component of electric field will be zero on both sides.

Consider an electromagnetic field $\left(\underline{H}^{A}, \underline{E}^{A}\right)$ that is generated by a current distribution $\underline{J}^{A}$ and another field $\left(\underline{H}^{B}, \underline{E}^{B}\right)$ that is generated by a current distribution $\underline{J}^{B}$. Providing that the fields share the same material properties within $V$, it can be 


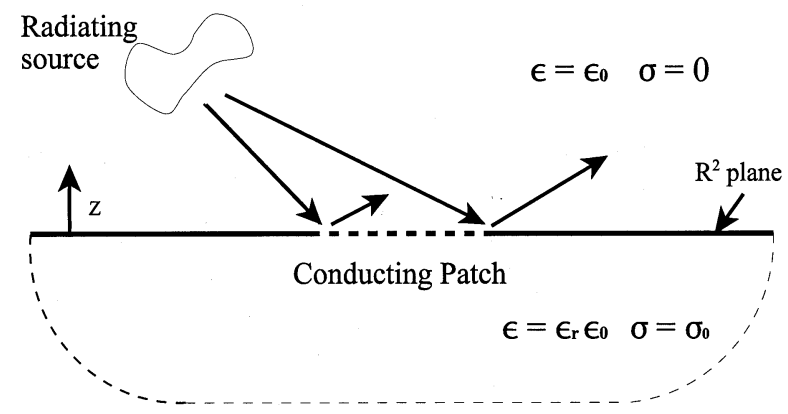

Fig. 1. Scattering by an antenna ground screen.

shown [10] that the time harmonic Maxwell equations imply the integral relation

$$
\begin{aligned}
\int_{S}\left(\underline{E}^{A} \times \underline{H}^{B}-\underline{E}^{B}\right. & \left.\times \underline{H}^{A}\right) \cdot d \underline{S}^{\prime} \\
& =\int_{V}\left(\underline{E}^{B} \cdot \underline{J}^{\prime A}-\underline{E}^{\prime A} \cdot \underline{J}^{B}\right) d V^{\prime}
\end{aligned}
$$

where a prime denotes a field that has been evaluated at point $\underline{r^{\prime}}$ and $S$ is the surface of volume $V$. Let $(\underline{H}, \underline{E})$ be the field due to a set of bounded sources in the upper half plane $\left(\underline{H}^{S}, \underline{E}^{S}\right)$ the field due to a unit current element $\underline{s}$ located at position $\underline{r}$. Then, substituting these fields for the $A$ and $B$ fields in (1)

$$
\left.-\int_{R^{2}} \underline{H}^{\prime S} \cdot\left(\underline{E}^{\prime} \times \underline{\hat{z}}\right)+\underline{E}^{\prime S} \cdot\left(\underline{H}^{\prime} \times \underline{\hat{z}}\right)\right) d S^{\prime}=C \underline{E} \cdot \underline{s}-\underline{E}^{i} \cdot \underline{s}
$$

with $V$ consisting of the upper half space $z>0 . \underline{E}^{i}$ is the field incident upon the $z=0$ plane (the field due to the sources of $(\underline{H}, \underline{E})$ acting alone) and $R^{2}$ is the boundary between the upper and lower half spaces with contributions from other boundaries vanishing due to the bounded nature of the sources. $(C=1$ when the current element is located at point $\underline{r}$ in the upper half plane and $C=0$ when it is located in the lower half plane.) Similarly, applying (1) to a volume consisting of the lower half space

$$
\left.\int_{R^{2}}{\underline{H^{\prime}}}^{T} \cdot\left(\underline{E}^{\prime} \times \underline{\hat{z}}\right)+\underline{E}^{\prime T} \cdot\left(\underline{H^{\prime}} \times \underline{\hat{z}}\right)\right) d S^{\prime}=(1-C) \underline{E} \cdot \underline{t}
$$

where $\left(\underline{H}^{T}, \underline{E}^{T}\right)$ is the field due to unit current element $\underline{t}$ located at point $\underline{r}$. Introducing the quantities $\underline{M}=\underline{E} \times \underline{\hat{z}}$ and $\underline{J}=\underline{\hat{z}} \times \underline{H}$, the previous equations can be written as

$$
\int_{R^{2}}\left(\underline{H}^{\prime S} \cdot \underline{M}^{\prime}-\underline{E}^{\prime S} \cdot \underline{J}^{\prime}\right) d S^{\prime}=-C \underline{E} \cdot \underline{s}+\underline{E}^{i} \cdot \underline{s}
$$

and

$$
\int_{R^{2}}\left({\underline{H^{\prime}}}^{T} \cdot \underline{M}^{\prime}-\underline{E}^{\prime T} \cdot \underline{J}^{\prime}\right) d S^{\prime}=(1-C) \underline{E} \cdot \underline{t} .
$$

The plane $R^{2}$ is a boundary on which the permittivity changes from a value $\varepsilon_{0}$ in the upper half space (note that the upper half space does not necessarily need to be a free space) to a value $\epsilon$ in the lower half space. As a consequence, field $\left(\underline{H}^{S}, \underline{E}^{S}\right)$ is calculated assuming a permittivity of $\varepsilon_{0}$ everywhere and $\left(\underline{H}^{T}, \underline{E}^{T}\right)$ is calculated assuming permittivity $\epsilon$ everywhere. The plane is also assumed to include some perfectly conducting patches that occupy region $G$ and for which the boundary conditions imply that $\underline{M}=0$ on both sides. Elsewhere on $R^{2}$, the boundary conditions will imply that $\underline{M}$ and $\underline{J}$ are continuous across the plane.

In the current work, we are interested in the behavior of the scattered fields at large distances from the sources $(r \rightarrow \infty)$ and so we will study (3) and (4) in this limit. The fields $\left({\underline{H^{\prime}}}^{T},{\underline{E^{\prime}}}^{T}\right.$ ) can be expanded for large $r$ as

$$
{\underline{E^{\prime}}}^{T} \approx j \eta \beta(\underline{\hat{r}} \cdot \underline{t} \underline{\hat{r}}-\underline{t}) \exp \left(j \beta \underline{\hat{r}} \cdot \underline{r}^{\prime}\right) \frac{\exp (-j \beta r)}{4 \pi r}
$$

and

$$
\underline{H}^{\prime T} \approx j \beta \underline{\hat{r}} \times \underline{t} \exp \left(j \beta \underline{\hat{r}} \cdot \underline{r}^{\prime}\right) \frac{\exp (-j \beta r)}{4 \pi r}
$$

and the fields $\left(\underline{H}^{\prime S}, \underline{E}^{\prime S}\right)$ as

$$
\underline{H}^{\prime S} \approx j \beta_{0} \underline{\hat{r}} \times \underline{s} \exp \left(j \beta_{0} \underline{\hat{r}} \cdot \underline{r}^{\prime}\right) \frac{\exp \left(-j \beta_{0} r\right)}{4 \pi r}
$$

and

$$
\underline{E}^{\prime S} \approx j \eta_{0} \beta_{0}(\underline{\hat{r}} \cdot \underline{s} \underline{\hat{r}}-\underline{s}) \exp \left(j \beta_{0} \underline{\hat{r}} \cdot \underline{r}^{\prime}\right) \frac{\exp \left(-j \beta_{0} r\right)}{4 \pi r}
$$

where $\underline{\hat{r}}$ is a unit vector in the radial direction. The sources of fields $(\underline{H}, \underline{E})$ will be assumed to be bounded and a finite distance from the conducting patches. Furthermore, the ground will be assumed to have sufficient conductivity for any surface waves to be rapidly attenuated. As a consequence, the scattered field sources will be effectively bounded and expansions (6)-(9) can be used to expand (4) and (5) in powers of $r^{-1}$. (The expansion will be valid for $r$ greater than $2 D^{2} / \lambda$ where $D$ is the greatest dimension of a region that contains both the sources and the patches.)

Let $\underline{\hat{r}}_{h}$ be the horizontal component of $\underline{\hat{\gamma}}$ (note that $\underline{\hat{\hat{r}}}=\underline{\hat{r}}_{h}+$ $\sqrt{1-\left|\underline{\hat{r}}_{h}\right|^{2}} \underline{\hat{z}}$ in the upper half plane) and define the interface fields $\underline{P}, \underline{Q}$, and $\underline{P}_{\Delta}$ by

$$
\begin{aligned}
& \underline{P}\left(\underline{\hat{r}}_{h}\right)=\int_{R^{2}} \exp \left(j \beta_{0} \underline{\hat{r}}_{h} \cdot \underline{r}^{\prime}\right) \underline{J}^{\prime} d S^{\prime} \\
& \underline{Q}\left(\underline{\hat{r}}_{h}\right)=\int_{R^{2}-G} \exp \left(j \beta_{0} \underline{\hat{r}}_{h} \cdot \underline{r}^{\prime}\right) \underline{M^{\prime}} d S^{\prime}
\end{aligned}
$$

and

$$
\underline{P}_{\Delta}\left(\underline{\hat{x}}_{h}\right)=\int_{G} \exp \left(j \beta_{0} \underline{\hat{r}}_{h} \cdot \underline{r}^{\prime}\right) \underline{J}_{\Delta}^{\prime} d S^{\prime}
$$

where $\underline{J}_{\Delta}$ is the difference in current between the lower and upper sides of the plates that constitute $G$. (Note that $P, Q$, and $\underline{P}_{\Delta}$ only have horizontal components). Furthermore, let $\underline{e}$ and $\underline{e}^{i}$ denote the fields $\underline{E}$ and $\underline{E}^{i}$ normalized by the factor $j \eta_{0} \beta_{0} \exp \left(j \beta_{0} r\right) / 4 \pi r$. On noting the boundary conditions that must be satisfied on the surface $R^{2}$, the leading order $\left(r^{-1}\right.$ term) of (4) and (5) will yield

$$
\begin{aligned}
& \frac{1}{\eta_{0}}(\underline{\tilde{r}} \times \underline{s}) \cdot \underline{Q}\left(\underline{\hat{r}}_{h}\right)-(\underline{\tilde{r}} \cdot \underline{s} \underline{\tilde{r}}-\underline{s}) \cdot \underline{P}\left(\underline{\hat{r}}_{h}\right)=\underline{e}^{i}(\underline{\widetilde{r}}) \cdot \underline{s} \\
& \frac{1}{\eta_{0}}(\underline{\hat{r}} \times \underline{s}) \cdot \underline{Q}\left(\underline{\hat{r}}_{h}\right)-(\underline{\hat{r}} \cdot \underline{s} \hat{\hat{r}}-\underline{s}) \cdot \underline{P}\left(\underline{\hat{r}}_{h}\right)=-\underline{e}(\underline{\hat{r}}) \cdot \underline{s}+\underline{e}^{i}(\underline{\hat{r}}) \cdot \underline{s}
\end{aligned}
$$

and

$$
\frac{1}{\eta}(\underline{\hat{r}} \times \underline{t}) \cdot \underline{Q}\left(\gamma \underline{\hat{r}}_{h}\right)-(\underline{\hat{\hat{r}}} \cdot \underline{\underline{\hat{r}}}-\underline{t}) \cdot\left(\underline{P}\left(\gamma \underline{\underline{\hat{r}}}_{h}\right)+\underline{P}_{\Delta}\left(\gamma \underline{\hat{\hat{r}}}_{h}\right)\right)=0
$$


where $\gamma=\beta / \beta_{0}$ and $\underline{\tilde{r}}=2 \underline{\hat{r}}_{h}-\underline{\hat{r}}$ is $\underline{\hat{r}}$ when evaluated at the conjugate point to $\underline{r}$.

It is possible to rescale (15) to yield

$$
\frac{1}{\eta}\left(\underline{\hat{r}}_{\gamma} \times \underline{t}\right) \cdot \underline{Q}\left(\underline{\hat{r}}_{h}\right)-\left(\underline{\hat{r}}_{\gamma} \cdot \underline{t}_{\gamma}-\underline{t}\right) \cdot\left(\underline{P}\left(\underline{\hat{r}}_{h}\right)+\underline{P}_{\Delta}\left(\underline{\hat{r}}_{h}\right)\right)=0
$$

where $\underline{\hat{r}}_{\gamma}=\underline{\hat{r}}_{h} \gamma^{-1}+\underline{\hat{z}} \sqrt{1-\hat{r}_{h}^{2} \gamma^{-2}}$ and $\hat{r}_{h}=\left|\underline{\hat{r}}_{h}\right|$. Since $\underline{s}$ and $\underline{t}$ are arbitrary unit vectors, (13) and (16) yield

$$
\begin{aligned}
\frac{1}{\eta} \underline{Q}\left(\underline{\hat{r}}_{h}\right) \times \underline{\hat{r}}_{\gamma}-\frac{1}{\gamma} \underline{\hat{r}}_{\gamma} \underline{\hat{r}}_{h} \cdot\left(\underline{P}\left(\underline{\hat{r}}_{h}\right)+\underline{P}_{\Delta}\left(\underline{\hat{r}}_{h}\right)\right) \\
+\left(\underline{P}\left(\underline{\hat{r}}_{h}\right)+\underline{P}_{\Delta}\left(\underline{\hat{r}}_{h}\right)\right)=0
\end{aligned}
$$

and

$$
\frac{1}{\eta_{0}} Q\left(\underline{\hat{r}}_{h}\right) \times \underline{\tilde{r}}-\underline{\tilde{r}}_{h} \cdot \underline{P}\left(\underline{\hat{r}}_{h}\right)+\underline{P}\left(\underline{\hat{r}}_{h}\right)=\underline{e}^{i}(\underline{\tilde{r}}) .
$$

Rearranging the components of (18)

$$
\frac{1}{\eta_{0}} \underline{Q}\left(\underline{\hat{r}}_{h}\right) \times \underline{\hat{r}}+\underline{\hat{r}}_{h} \cdot \underline{P}\left(\underline{\hat{r}}_{h}\right)-\underline{P}\left(\underline{\hat{r}}_{h}\right)=-\underline{\widetilde{e}}^{i}(\underline{\widetilde{r}})
$$

where $\underline{\tilde{e}}^{i}=2 \underline{e}_{h}^{i}-\underline{e}^{i}$. From (14), the desired quantity ( $\underline{e}$ in the upper half space) is related to $\underline{P}$ and $\underline{Q}$ through

$$
\frac{1}{\eta_{0}} \underline{Q}\left(\underline{r}_{h}\right) \times \underline{\hat{r}}-\underline{\hat{r} \hat{r}} \cdot \underline{P}\left(\underline{r}_{h}\right)+\underline{P}\left(\underline{r}_{h}\right)=-\underline{e}(\underline{\hat{r}})+\underline{e}^{i}(\underline{\hat{r}})
$$

and, on eliminating $\underline{Q} \wedge \underline{\hat{r}}$ by means of (19)

$$
-2 \underline{\hat{r}} \underline{\hat{r}}_{h} \cdot \underline{P}\left(\underline{\hat{r}}_{h}\right)+2 \underline{P}\left(\underline{\hat{r}}_{h}\right)=-\underline{e}(\underline{\hat{r}})+\underline{e}^{i}(\underline{\hat{r}})+\underline{\widetilde{e}}^{i}(\underline{\tilde{r}}) .
$$

Consequently, $\underline{e}$ can be expressed totally in terms of $\underline{e}^{i}$ and $\underline{P}$ (note that $\underline{e} \cdot \underline{\hat{r}}=0$ ).

It remains to find a relationship between $\underline{e}^{i}$ and $\underline{P}$. Consider the horizontal components of (17) and (19)

$$
\begin{array}{r}
\frac{\sqrt{1-\hat{r}_{h}^{2} \gamma^{-2}}}{\eta} \underline{Q}\left(\underline{\hat{r}}_{h}\right) \times \underline{\hat{z}}-\gamma^{-2} \underline{\hat{r}}_{h} \underline{\hat{r}}_{h} \cdot\left(\underline{P}\left(\underline{\hat{r}}_{h}\right)+\underline{P}_{\Delta}\left(\underline{\hat{r}}_{h}\right)\right) \\
+\left(\underline{P}\left(\underline{\hat{r}}_{h}\right)+\underline{P}_{\Delta}\left(\underline{\hat{r}}_{h}\right)\right)=0
\end{array}
$$

and

$$
\frac{\sqrt{1-\hat{r}_{h}^{2}}}{\eta_{0}} \underline{Q}\left(\underline{\hat{r}}_{h}\right) \times \underline{\hat{z}}+\underline{\hat{r}}_{h} \underline{\hat{r}}_{h} \cdot \underline{P}\left(\underline{\hat{r}}_{h}\right)-\underline{P}\left(\underline{\hat{r}}_{h}\right)=-\underline{e}_{h}^{i}(\underline{\widetilde{r}}) .
$$

From (22) and (23)

$$
\begin{array}{r}
S \cdot \underline{P}\left(\underline{\hat{r}}_{h}\right)=-\frac{\sqrt{1-\hat{r}_{h}^{2}}}{\eta_{0}}\left(\gamma^{-2} \underline{\hat{r}}_{h} \underline{\hat{r}}_{h} \cdot \underline{P}_{\Delta}\left(\underline{\hat{r}}_{h}\right)-\underline{P}_{\Delta}\left(\underline{\hat{r}}_{h}\right)\right) \\
-\frac{\sqrt{1-\hat{r}_{h}^{2} \gamma^{-2}}}{\eta} \underline{e}_{h}^{i}(\underline{\tilde{r}})
\end{array}
$$

and

$$
\begin{aligned}
& S \cdot\left(Q\left(\underline{\hat{r}}_{h}\right) \times \underline{\hat{z}}\right)=-\gamma^{-2}\left(1-\hat{r}_{h}^{2}\right) \underline{\hat{r}}_{h} \underline{\hat{r}}_{h} \cdot \underline{P}_{\Delta}\left(\underline{\hat{r}}_{h}\right) \\
& -\underline{\hat{r}}_{h} \underline{\hat{r}}_{h} \cdot \underline{P}_{\Delta}\left(\underline{\hat{r}}_{h}\right)+\underline{P}_{\Delta}\left(\underline{\hat{r}}_{h}\right)-\gamma^{-2} \underline{\hat{r}}_{h} \underline{\hat{r}}_{h} \cdot \underline{e}_{h}^{i}(\underline{\hat{r}})+\underline{e}_{h}^{i}(\underline{\hat{r}})
\end{aligned}
$$

where $S$ is the dyadic operator

$$
S=\frac{\sqrt{1-\hat{r}_{h}^{2} \gamma^{-2}}}{\eta}\left(\underline{\hat{r}}_{h} \underline{\hat{r}}_{h}-I\right)+\frac{\sqrt{1-\hat{r}_{h}^{2}}}{\eta_{0}}\left(\gamma^{-2} \underline{\hat{r}}_{h} \underline{\hat{r}}_{h}-I\right)
$$

and $I$ is the unit dyad. For $\left|\underline{\hat{r}}_{h}\right|<1$, the inverse operator (see (27) at the bottom of the page) can be used to derive expressions for $\underline{P}$ and $\underline{Q} \wedge \underline{\hat{z}}$ in terms of $P_{\Delta}$ and $\underline{e}_{h}^{i}$. (Note that the case where $\left|\underline{\hat{r}}_{h}\right|=1$ will need to be treated as a limit. This limit will exist providing that the ground is sufficiently lossy to remove surface waves from the far field.) For the special case of no patches $\left(\underline{P}_{\Delta}=0\right),(24)$ will yield (28) (as shown at the bottom of the page), where $\hat{r}_{z}=\sqrt{1-\hat{r}_{h}^{2}}$ and $\hat{r}_{z}^{\gamma}=\sqrt{1-\hat{r}_{h}^{2} \gamma^{-2}}$. The scattered fields will follow directly from (21).

\section{AN IMPORTANT LIMIT}

Unfortunately, when there are conducting patches, the calculations are far more complex. It will be noted that, in most practical situations, $\gamma \gg 1$ and $\eta \ll \eta_{0}$. In this case, ignoring terms in $\gamma^{-2},(24)$ yields the expression

$$
\begin{aligned}
& \underline{P}\left(\underline{\hat{r}}_{h}\right)=\left(\eta_{0} \underline{e}_{h}^{i}(\underline{\tilde{r}})-\eta \underline{P}_{\Delta}\left(\underline{\hat{r}}_{h}\right) \hat{r}_{z}\right. \\
& \left.\quad+\eta_{0} \underline{\hat{r}}_{h} \frac{\eta_{0} \underline{\hat{r}}_{h} \cdot \underline{e}_{h}^{i}(\underline{\tilde{r}})-\eta \underline{\hat{r}}_{h} \cdot \underline{P}_{\Delta}\left(\underline{\hat{r}}_{h}\right) \hat{r}_{z}}{\eta \hat{r}_{z}+\eta_{0} \hat{r}_{z}^{2}}\right) \frac{1}{\eta \hat{r}_{z}+\eta_{0}} .
\end{aligned}
$$

This is exactly the expression that would result if an impedance boundary condition had been used to represent the boundary [11]. It still remains, however, to determine $\underline{P}_{\Delta}$. From (25), using the inverse of $S$ and ignoring terms in $\gamma^{-2}$

$\frac{1}{\eta} \underline{Q}\left(\underline{\hat{r}}_{h}\right) \times \underline{\hat{z}}+\underline{P}_{\Delta}\left(\underline{\hat{r}}_{h}\right)=\frac{\left(\underline{\hat{z}} \times \underline{\hat{r}}_{h}\right) \cdot \underline{e}_{h}^{i}(\underline{\widetilde{r}})\left(\underline{\hat{z}} \times \underline{\hat{r}}_{h}\right)-\underline{e}_{h}^{i}(\underline{\tilde{r}})}{\hat{r}_{z}^{2}}$.

$$
S^{-1}=\eta \eta_{0} \frac{\eta_{0} \sqrt{1-\hat{r}_{h}^{2} \gamma^{-2}}\left(\left(\underline{\hat{z}} \times \underline{\hat{r}}_{h}\right)\left(\underline{\underline{\hat{z}}} \times \underline{\hat{r}}_{h}\right)-I\right)+\eta \sqrt{1-\hat{r}_{h}^{2}}\left(\gamma^{-2}\left(\underline{\hat{z}} \times \underline{\hat{r}}_{h}\right)\left(\underline{\underline{\hat{z}}} \times \underline{\hat{r}}_{h}\right)-I\right)}{\sqrt{1-\hat{r}_{h}^{2}} \sqrt{1-\hat{r}_{h}^{2} \gamma^{-2}}\left(\eta_{0} \sqrt{1-\hat{r}_{h}^{2}}+\eta \sqrt{1-\hat{r}_{h}^{2} \gamma^{-2}}\right)\left(\eta \sqrt{1-\hat{r}_{h}^{2}}+\eta_{0} \sqrt{1-\hat{r}_{h}^{2} \gamma^{-2}}\right)}
$$

$$
\frac{\underline{P}\left(\underline{\hat{r}}_{h}\right)}{\eta_{0}}=\frac{\eta_{0} \hat{r}_{z}^{\gamma}\left(\underline{e}_{h}^{i}(\underline{\widetilde{r}})-\left(\underline{\hat{z}} \times \underline{\hat{r}}_{h}\right)\left(\underline{\hat{z}} \times \underline{\hat{r}}_{h}\right) \cdot \underline{e}_{h}^{i}(\underline{\widetilde{r}})\right)+\eta \hat{r}_{z}\left(\underline{e}_{h}^{i}(\underline{\widetilde{r}})-\gamma^{-2}\left(\underline{\hat{z}} \times \underline{\hat{r}}_{h}\right)\left(\underline{\hat{z}}^{\prime} \times \underline{\hat{r}}_{h}\right) \cdot \underline{e}_{h}^{i}(\underline{\widetilde{r}})\right)}{\hat{r}_{z}\left(\eta_{0} \hat{r}_{z}+\eta \hat{r}_{z}^{\gamma}\right)\left(\eta \hat{r}_{z}+\eta_{0} \hat{r}_{z}^{\gamma}\right)}
$$


Now consider the identity

$\int_{G} A\left(\underline{r}^{\prime}\right) \exp \left(j \beta \underline{\hat{r}}_{h} \cdot \underline{\hat{r}}^{\prime}\right) d x^{\prime} d y^{\prime}=$

$\int_{R^{2}} K\left(\underline{\hat{r}}_{h}-\underline{r}^{\prime \prime}\right)\left(\int_{R^{2}} A\left(\underline{r}^{\prime \prime \prime}\right) \exp \left(j \beta \underline{r}^{\prime \prime} \cdot \underline{\hat{r}}^{\prime \prime \prime}\right) d x^{\prime \prime \prime} d y^{\prime \prime \prime}\right) d x^{\prime \prime} d y^{\prime \prime}$

where

$$
K(\underline{\alpha})=\frac{\beta^{2}}{4 \pi^{2}} \int_{G} \exp \left(j \beta \underline{r^{\prime}} \cdot \underline{\alpha}\right) d S^{\prime} .
$$

This can be derived by Fourier transform techniques and, for a rectangular $G$ with sides aligned to the coordinate axes

$$
\begin{aligned}
K(\underline{\alpha})=\frac{1}{\alpha_{x} \alpha_{y} \pi^{2}} \exp \left(j \beta \underline{r}_{C} \cdot \underline{\alpha}\right) \\
\quad \sin \cdot\left(\frac{\beta \alpha_{x} L_{x}}{2}\right) \sin \left(\frac{\beta \alpha_{y} L_{y}}{2}\right)
\end{aligned}
$$

when the center is located at $\underline{r}_{C}$ and the sides have lengths $L_{x}$ and $L_{y}$, respectively. (More complex shapes can be constructed from a collection of rectangles.) Noting that the integrand of the integral forming $Q$ is zero inside region $G$, and that of $\underline{P}_{\Delta}$ is zero outside $G$, (33) can be applied to (30) to yield

$$
\underline{P}_{\Delta}\left(\underline{\hat{r}}_{h}\right)=\int_{R^{2}} K\left(\underline{\hat{r}}_{h}-\underline{r}^{\prime \prime}\right) \underline{R}\left(\underline{r}^{\prime \prime}\right) d x^{\prime \prime} d y^{\prime \prime}
$$

where

$$
\underline{R}\left(\underline{\hat{r}}_{h}\right)=\frac{\left(\underline{\hat{z}} \times \underline{\hat{r}}_{h}\right) \cdot \underline{e}_{h}^{i}(\underline{\widetilde{r}})\left(\underline{\hat{z}} \times \underline{\hat{r}}_{h}\right)-\underline{e}_{h}^{i}(\underline{\widetilde{r}})}{\hat{r}_{z}^{2}} .
$$

These expressions provide the required relationship between $\underline{P}_{\Delta}$ and $\underline{e}^{i}$. It will be noted that the same expressions would also result if $\underline{P}_{\Delta}$ were calculated on the basis of currents induced in an infinite conducting plane (which is equivalent to the physical optics approximation) [11]. In general, it is possible to use the inverse of $S$ to isolate $Q \wedge \underline{\hat{z}}$ in (25) and to apply of integral identity (31). There will now result in an integral equation for $\underline{P}_{\Delta}$ which can then be solved by numerical techniques. The leading order solution provided by (34) and (35) is, however, adequate for most purposes.

\section{SOME EXAMPLES}

The considerations of Section III have been implemented as a computer program that takes, as its input, the currents on an antenna when simulated over an infinite perfectly conducting plane. It produces, as its output, the gain pattern of the same antenna when placed over a finitely conducting plane that contains perfectly conducting patches. As a sample application of the program, consider a quarter wave monopole placed on a ground screen. Fig. 2 shows the gain pattern that results when the monopole is placed just inside a rectangular screen that has dimensions of $10 \lambda$ by $4 \lambda$ ( $\lambda$ is the wavelength). The pattern to the left is the response in the direction that contains majority of

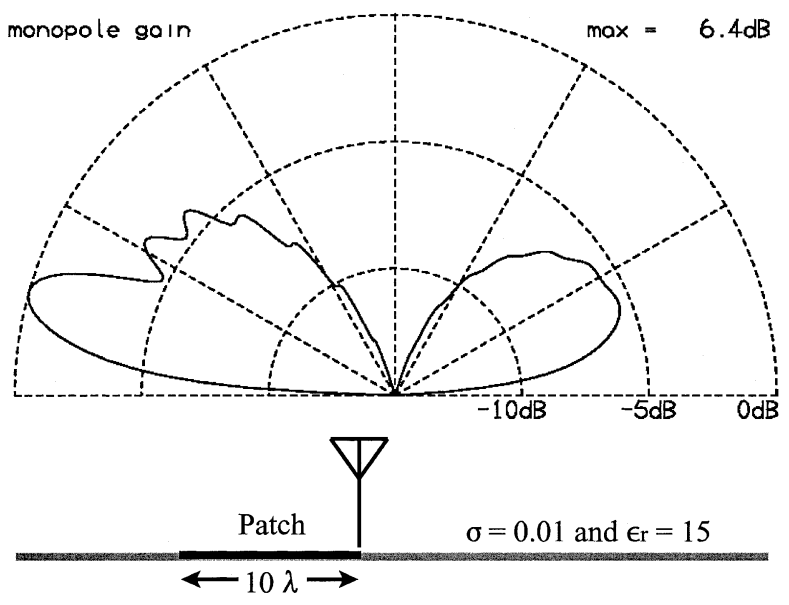

Fig. 2. Monopole over asymmetric ground screen ( $\sigma$ in units of $\mathrm{S} / \mathrm{m}$ ).
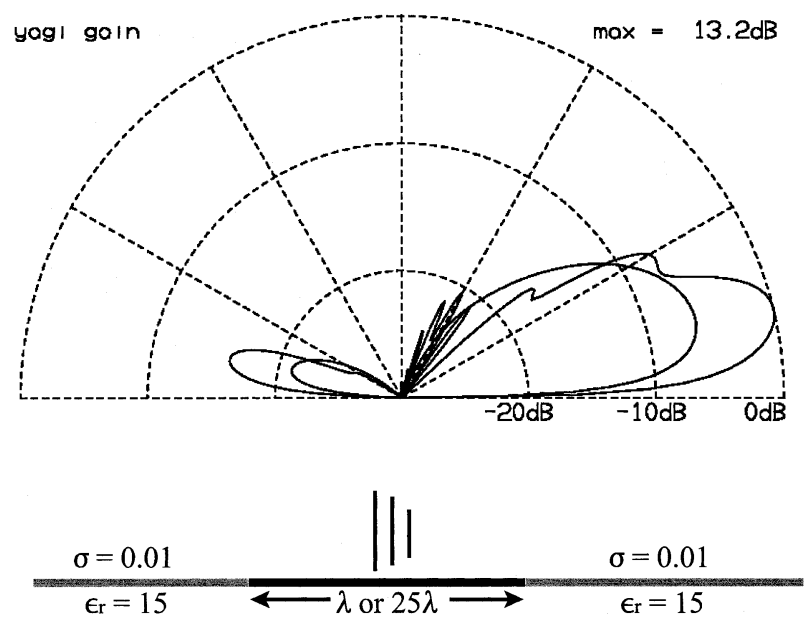

Fig. 3. Yagi over different ground screens ( $\sigma$ in units of $\mathrm{S} / \mathrm{m}$ ).

the screen. It will be noted that the gain has a good response at low elevations and exhibits the characteristic ripples of edge diffraction at high elevations. To the right, there is a much reduced pattern with poor low-elevation response. Such behavior is to be expected since there is very little screen in this direction (less than half a wavelength) and so a large amount of energy will be dissipated in the ground. Sinnott [7] has considered the problem of ground screens by means of compensation theorem techniques. In this work, the ground screens extended from the antenna (a short dipole) by up to $8 \lambda$. The current techniques have been used to simulate the Sinnott results and have been found to be within $0.5 \mathrm{~dB}$ in all cases. A more demanding test of the current procedure is provided by a three-element vertical yagi that is placed over a finite ground screen. Fig. 3 shows the superimposed gain patterns for square screens of side $\lambda$ and $25 \lambda$, respectively, (the lower value of maximum gain corresponds to screen with side $\lambda$ ). It will be noted that low-elevation response improves as screen size increases, but the cost is strong edge diffraction ripples at the higher elevations.

Although a perfectly conducting ground screen is a good approximation for many situations, there are some problems for which the finite conductivity can be important. The considera- 

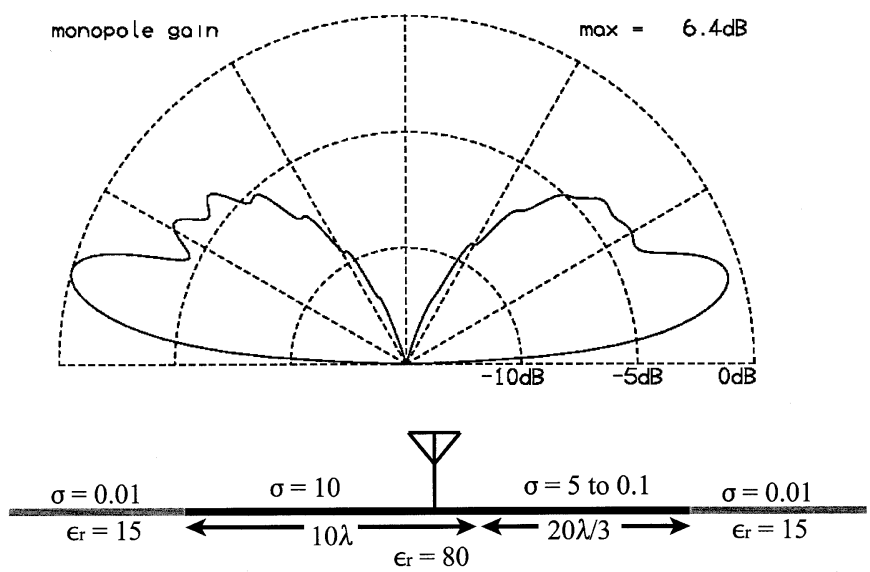

Fig. 4. Monopole over spatially varying ground screen $(\sigma$ in units of $\mathrm{S} / \mathrm{m})$.

tions of Section II can extended to this case and the same formulas can be used providing that $K$ is now calculated from

$$
K(\underline{\alpha})=\frac{\beta^{2}}{4 \pi^{2}} \int_{G}\left(1-\frac{\eta_{G}\left(\underline{r}^{\prime}\right)}{\eta}\right) \exp \left(j \beta \underline{r^{\prime}} \cdot \underline{\alpha}\right) d S^{\prime}
$$

where $\eta_{G}$ is the distribution of surface impedance within the patches [11]. An interesting application of this extension is to be found in the improvement of ground screens. It will be noted that a major downside of increasing the size of a screen is the appearance of strong ripples in the gain pattern at higher elevations. As with the Gibbs phenomena, this effect is a result of a sudden change in conditions. The Gibbs phenomena can be moderated by replacing sudden changes with smoother transitions (windowing in signal processing terms) and this would suggest a more gentle transition from the strong conductivity of the screen to that of the surrounds. In practical terms, the screen will be made out of a mesh and this can be progressively thinned out toward the edges. Fig. 4 shows the radiation pattern of a quarter wave monopole that has been placed at the center of a rectangular screen. The surface impedance of the screen decreases to the right (the reduction achieved through a series of equal sized subpatches) but, to the left, is constant until an abrupt change at the edge of the screen. As expected, the effect of the more gentle adjustment is a smoothing out of the edge diffraction ripples. In addition, the low-elevation response and overall gain are little affected. It is possible that further improvements in the pattern would follow from the use of a more continuous impedance profile.

\section{CONCLUSION}

Equations have been developed that connect the scattered fields, caused by conducting patches, to the radiative behavior of the field sources acting alone. In the most realistic limit (a ground with relatively large dielectric constant), the equations can be solved and there results a straightforward technique for investigating scattering by an imperfect ground. Importantly, the method does not need to calculate ground currents and, hence, avoids the use of a physical optics approximation on the ground screen. Indeed, the calculations only require the far field behavior of the incident fields to be known. The method has been demonstrated through its application to some nontrivial antenna calculations involving ground screens. In particular, it has been shown that, when the ground screen impedance smoothly blends into that of its surrounds, the effects of edge diffraction can be substantially reduced.

\section{ACKNOWLEDGMENT}

The author would like to thank the referees for helpful comments.

\section{REFERENCES}

[1] A. N. Sommerfeld, "The propagation of waves in wireless telegraphy," Ann. Phys., ser. 4, vol. 28, pp. 665-736, 1909.

[2] - "The propagation of waves in wireless telegraphy," Ann. Phys., ser. 4, vol. 81, pp. 1135-1153, 1926.

[3] K. A. Norton, "Propagation of radio waves over the surface of the earth and upper atmosphere," Proc. IRE, vol. 24, pp. 1367-1387, 1936.

[4] R. W. P. King, "New formulas for the electromagnetic field of a vertical electric dipole in a dielectric or conducting half space near its horizontal interface," J. Appl. Phys., vol. 53, pp. 8476-8482, 1984.

[5] G. J. Burke and A. J. Poggio, "Numerical electromagnetic code (NEC)-Method of moments," Lawrence Livermore Lab., Livermore, CA, Tech. Rep. UCID18834, 1981.

[6] J. R. Wait, "The theory of an antenna over an inhomogeneous ground plane," in Electromagnetic Theory of Antennas, E. C. Jordan, Ed. New York: Pergamon, 1963, pp. 1079-1098.

[7] D. H. Sinnott, "Elevation patterns of HF antennas over ground screens," in Conv. Dig., IREE Conv., Sydney, Australia, 1983, pp. 604-606.

[8] C. J. Coleman, "On the application of the extinction theorem to some antenna problems," IEE Proc.-Microw. Antennas Propag., vol. 143, pp. $471-474,1996$.

[9] _ - "The effect of an imperfectly conducting plane upon an incident electromagnetic field," IEEE Trans. Antennas Propagat., vol. 45, pp. $1445-1450,1997$.

[10] G. D. Monteath, Applications of the Electromagnetic Reciprocity Principle. New York: Pergamon, 1973.

[11] C. J. Colman, "The effect of inhomogeneous ground upon HF antennas," in Proc. 1998 IEEE Antennas and Propagation Society Int. Symp., 1998, pp. $1462-1465$.

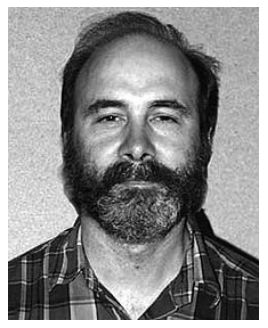

Chris J. Coleman (M'96) received the B.Sc. and Ph.D. degrees in mathematics from Imperial College, London University, London, U.K., in 1971 and 1974, respectively, and the B.Eng. degree in electrical and electronic engineering from the University of Adelaide, Adelaide, SA, Australia, in 2000.

From 1974 to 1990, he held research and teaching positions at Imperial College, the University of Wales at Aberystwyth, U.K, the University of Nottingham, Nottingham, U.K. and the University of Wollongong, Wollongong, Australia. In 1990, he joined the Defence Science and Technology Organization of Australia to work on the Jindalee over the horizon radar project. At the beginning of 1999, he joined the academic staff of the Electrical and Electronic Engineering Department, the University of Adelaide, where he now carries out teaching and research in the areas of antennas and propagation. 